\title{
Wide range scanning probe microscopy for probing mechanical effects on cellular function*
}

\author{
Takeomi Mizutani, Hisashi Haga, Kosaku Kato, and Kazushige Kawabata \\ Division of Biological Sciences, Graduate School of Science, Hokkaido University, Sapporo, Japan
}

\begin{abstract}
Summary. Scanning probe microscopy (SPM) provides a range of strategies for studying biological phenomena due to its ability to image surfaces under liquids. However, some cellular events, such as cell migration, exceed the maximum measurable range of SPM. Recently, we have developed a wide range scanning probe microscope (WRSPM) to investigate cellular events which exceed the range of the conventional SPM. In this review, we introduce the instrumentation of the WR-SPM, which can measure a sample for $400 \mu \mathrm{m}$ in the $x y$ directions and $23 \mu \mathrm{m}$ in the $z$ direction. We then show the application of the WRSPM to studies of the stiffness response of epithelial cells to an external loading force and demonstrat that the stiffness of the epithelial cells increases under stretched conditions. We also showed the results on the mesh structure on the surface of a melanoma cell as well as the regulatory mechanism of the cellular contractile force by the combined use of topographical and mechanical modes of the WR-SPM. These findings indicate that the WR-SPM is very useful for studying the functions of a cell in relation to the surface structure and mechanical properties of that cell.
\end{abstract}

Received January 5, 2009

*This work is partly supported by CASIO Science Promotion Foundation and by the Foundation Advanced Technology Institute for T.M., by a Grant-in-Aid for JSPS Fellows (20 .3710 for K. Kato), and by a Grant-in-Aid for Scientific Research from the Japan Society for the Promotion of Science (18340122 for K. Kawabata).

*Address for correspondence: Dr Takeomi Mizutani, Division of Biological Sciences, Graduate School of Science, Hokkaido University, Sapporo 060-0810, Japan

Tel: +81-11-706-3810, Fax: +81-11-706-3810

E-mail: mizutani@sci.hokudai.ac.jp

\section{Introduction}

Mechanical properties of living cells and physical parameters in their living condition play important roles in the expression of cellular functions, such as cell migration, cell division, and cell shaping. For example, the cellular contractile force induces cell migration by pushing the cell body forward (Mierke et al., 2008). The rigid cortex of the cellular surface $r$ maintains the shape of the cell against hydrodynamic forces (Mitchison et al., 2008). Moreover, stiffness of the substrate to which cells adhere, affects the direction of cell migration (Lo et al., 2000), differentiation (Engler et al., 2006), and cell division (Yeung et al., 2005). Although molecular based signal cascades have been proposed for each function of the cell, the details are not fully understood. A possible means to elucidate the effect of these cellular and/or extracellular mechanics on cellular functions is to measure the force and stiffness tempo-spatially and subseguently to compare the force maps on the cell with the molecular based cascades.

In various fields of science and technology, the scanning probe microscope (SPM) is widely known as a powerful instrument which can be used to visualize the morphology of samples at a high resolution on the nanometer scale. In addition, as the SPM can be combined with fluorescence microscopy, the combination is frequently used to study morphological changes of a sample by molecule-molecule interaction or intermolecular force. Currently, many types of SPM are used to measure the adhesive force, frictional force, and/or electrostatic properties of various types of biomaterials, including lipid membranes, proteins, and other biopolymers. In particular, the mechanical mode of SPM - which was developed for visualizing the spatial distribution of local viscoelasticity-has been used to observe the mechanical properties of living cells in 
liquids. By the combined use of SPM and fluorescence microscopy, we have shown that stiff fibrous structures measured by the mechanical mode correspond to the distribution of actin filaments in a fibroblast (Haga et al., 2000). In addition, stiff fibrous structures reflect the actomyosin-based cellular contractile force (Nagayama et al., 2004).

Although the mechanical mode-SPM is a powerful tool to investigate cellular function, most living phenomena at the cellular level exceed the scanning ranges of the conventional SPM that are usually $100 \mu \mathrm{m}$ in the $x y$ directions and $10 \mu \mathrm{m}$ in the $z$ direction. For example, tensional mechanisms of cell migration, or cellular contractile forces acting on the tissue-like material which comprises cells and the extracellular matrix (ECM), exceed SPM scanning ranges. Development of a SPM which can be used to measure in the submillimeter range is essential for the investigation of living phenomena at the cellular level. In this review, we will introduce a wide range scanning probe microscope (WR-SPM) which is specialized to measure a sample for $400 \mu \mathrm{m}$ in the $x y$ directions and $23 \mu \mathrm{m}$ in the $z$ direction. We will then show our application of WR-SPM to studies on the functions of the cell.

\section{Instrumentation}

We developed a wide range SPM (WR-SPM) based on a commercial SPM (Mizutani et al., 2004b). In order to expand the area of measurement, two conventional cylindrical piezotranslators (PZT) were jointed in tandem (Fig. 1a). The specification of the PZTs are as follows: The lower PZT has an $x y$-range of $170 \mu \mathrm{m}$ square and a $z$-range of $6 \mu \mathrm{m}$, and the upper one has a $z$-range of $17 \mu \mathrm{m}$. In this system, we only used the upper PZT for the movement in $\mathrm{z}$ direction, while the lower PZT was used for the movement in both $\mathrm{xy}$ and $\mathrm{z}$ directions. This achieved maximum scan ranges of $400 \mu \mathrm{m}$ square in the $x y$-plane and $23 \mu \mathrm{m}$ along the $z$-axis.

Reliability of the WR-SPM for lateral scanning was checked by obtaining topographic images of a standard pattern with $12 \mu \mathrm{m}$ square grids at various scanning frequencies. Figures $1 \mathrm{~b}-\mathrm{d}$ show typical images obtained with the constant force mode at four different scanning frequencies. Steady images were obtained below $1.0 \mathrm{~Hz}$ (Fig. 1b). However, some improper periodic noises began to appear over $1.5 \mathrm{~Hz}$ (marked by arrows in Fig. 1c). The periodic noises completely covered a whole image over $3.0 \mathrm{~Hz}$, and the square grids were not recognized (Fig. 1d). We thus decided that the reliable frequency for the lateral scanning in the WR-SPM is below $1.0 \mathrm{~Hz}$. The quantitativeness of the WR-SPM imaging was evaluated by the measurement of the standard pattern. Figure 1e shows a typical topography scanned with the maximum $x y$-range of the WR-SPM at the lateral frequency of 0.25 $\mathrm{Hz}$. Because 34 grids are observed in each side of the topography. the maximum scanning area of the WR-SPM achieves about $400 \mu \mathrm{m}$ square To asswss specification in the z-range, grids with $12 \mu \mathrm{m}$ square were observed (Fig.1f), and the cross-section of a grid line (marked by a white line in Fig. 1f) is shown in Figure 1g. The obtained height of the grid $(110 \mathrm{~nm})$ deviated somewhat from the nominal value of $100 \mathrm{~nm}$. In consideration of this deviation, the height data and force distance curves were accordingly calibrated linearly.

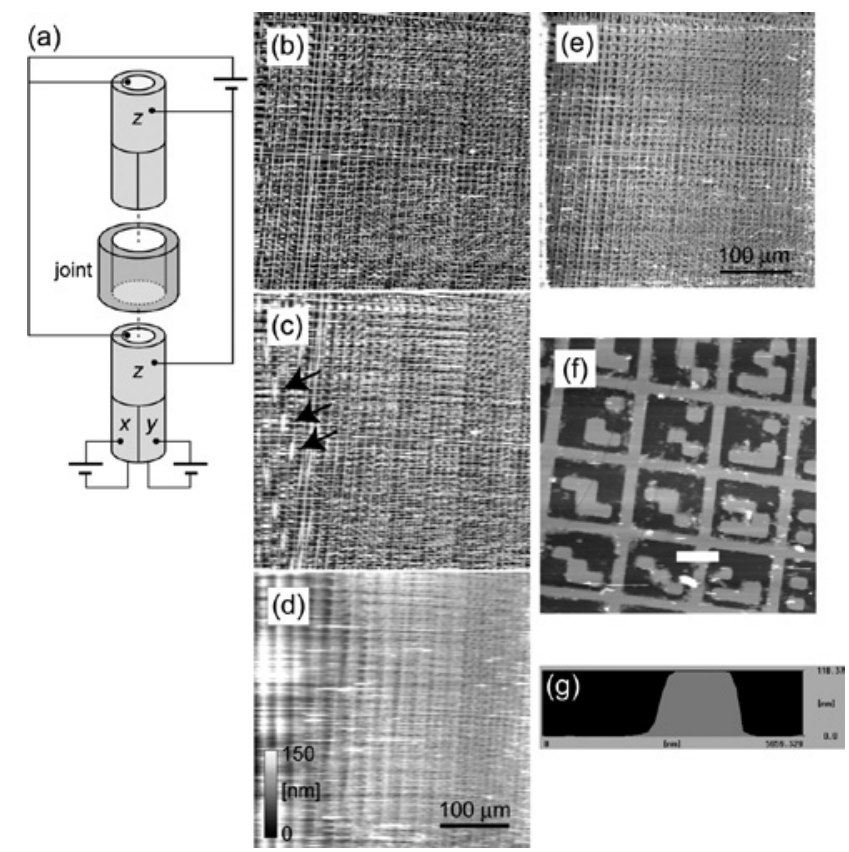

Fig. 1. a: Schematic drawing of a WR-SPM scanner. Two piezotranslators (PZT) were jointed with a cylindrical holder in tandem. Both $z$-axis electrodes were connected in parallel. bd: Influence of lateral scanning frequency on the topographies measured by the WR-SPM. A silicon substrate with square grids was measured at different scanning frequencies. Each image was measured at the lateral frequency of $1.0 \mathrm{~Hz}(\mathbf{b}), 1.5$ $\mathrm{Hz}(\mathbf{c})$, and $3.0 \mathrm{~Hz}(\mathbf{d})$. e: Topography of a silicon substrate carved square grids in the maximum $x y$-range of the WR-SPM. f: Topography of the same pattern in a $40 \mu \mathrm{m}$ square range scanned with the WR-SPM. g: A cross-sectional image of a grid line shown along the white line in f. Scanning frequency was $0.25 \mathrm{~Hz}$ for all measurements. Reproduced with permission from Mizutani et al. (2004b). Jpn J Appl Phys 43: 4525-4528. 
Fig. 2. Topography and stiffness map of epithelial cells. Epithelial cells (MDCK cell) were cultured on a glass substrate and measured with WR-SPM. More than 10 cells existed in the colony. Stiffness of the cell in the colony is not uniform. Bar $=50 \mu \mathrm{m}$
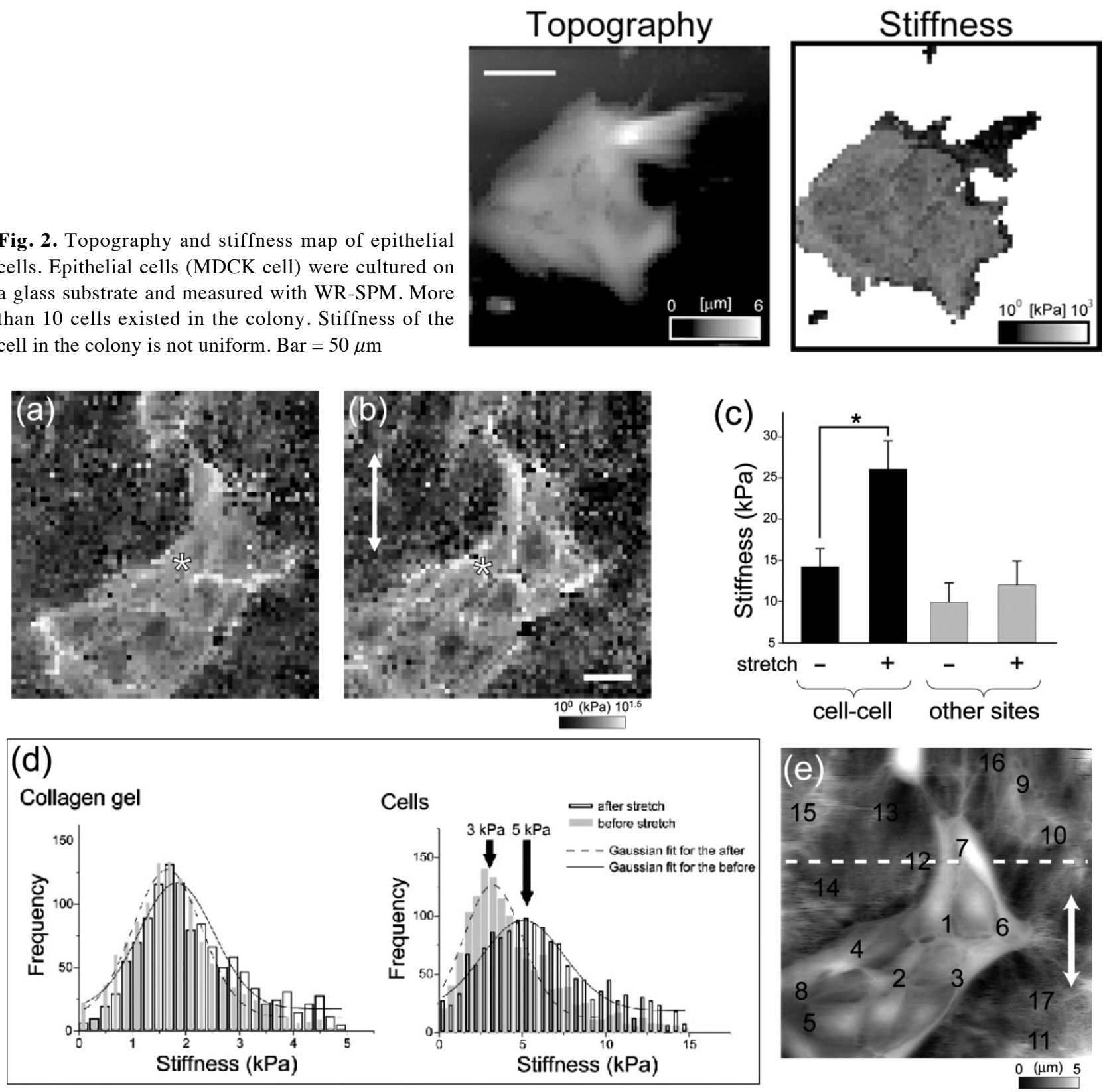

Fig. 3. a, b: Change in a spatial stiffness map due to stretch. Epithelial cells cultured on a collagen gel were stretched and stiffness maps were measured before (a) and after (b) the stretch. The arrows indicate the direction of application of the stretch. The bar represents $50 \mu \mathrm{m}$. c: Statistical analysis for cellular stiffness change depending on the area on the colony. The stiffness at junctional sites (representative is marked as an asterisk in $\mathbf{a}$ and $\mathbf{b}$ ) and other regions of the cells was averaged and statistical changes in the average stiffness were examined using Student's $t$ test. These data were presented as mean \pm SEM from about 100 pixel points of each image $\left({ }^{*} P<0.05\right)$. d: Histograms of stiffness distribution of collagen gels and cells before and after the uniaxial stretch. Each data was fitted to a Gaussian function. e: Change in the topographic images of epithelial cells under the stretch. The cells on the surface of the collagen gel were measured by WR-SPM before and after the stretch. The direction of the uniaxial stretch is represented by a set of arrows. Some points on the image have been numbered to analyze strain fields. The dashed line defines the origin of the stretch. Reproduced with permission from Mizutani et al. (2007). Acta Biomaterialia 3: 485493. 


\section{Stiffness response of epithelial cells to external loading force by WR-SPM}

Mechanical loading plays an important role in the meintenance of tissues and organs. For example, bones, muscles, and tendons require moderate external loading to maintain their mechanical strength (Benjamin and Hillen, 2003; Kljaer, 2004). The external loading force is transmitted into the tissues in a heterogeneous manner based on the morphology mechanical properties of the tissue. Therefore, the responses from each region in the tissue to the external loading force will be spatially different. The heterogeneity in the transmission of the external loading force into the tissue is essential for the maintenance of the tissue structure (vanEijden et al., 1996; Wiskott and Belser, 1999). At the present time, it is difficult to examine the biomechanical effects of the external loading force on the tissues because there is no

Table 1. Strain fields on the collagen gel and the epithelial cells analyzed from Figure $3 \mathrm{e}$

\begin{tabular}{|c|c|c|c|}
\hline & Number & Strain $(\%)$ & \\
\hline \multicolumn{4}{|l|}{ Cells } \\
\hline & 1 & 4.5 & \\
\hline & 2 & 5.2 & \\
\hline & 3 & 6.3 & \\
\hline & 4 & 5.7 & \\
\hline & 5 & 6.2 & \\
\hline & 6 & 4.8 & \\
\hline & 7 & 5.2 & \\
\hline & 8 & 6.8 & \\
\hline \multicolumn{4}{|l|}{ Collagen gel } \\
\hline & 9 & 5.3 & \\
\hline & 10 & 4.8 & \\
\hline & 11 & 5.1 & \\
\hline & 12 & 0 & $\begin{array}{l}\text { (defined as origen of } \\
\text { the stretch) }\end{array}$ \\
\hline & 13 & 5.5 & \\
\hline & 14 & 4.7 & \\
\hline & 15 & 5.2 & \\
\hline & 16 & 5.4 & \\
\hline & 17 & 4.8 & \\
\hline
\end{tabular}

Reproduced with permission from Mizutani et al. (2007), Acta Biomaterial 3: 489-493 apparatus that can measure the local surface strain and local stiffness simultaneously.

We recently constructed a device to stretch tissuelike materials and measured a stiffness map and the local surface strain of the cell using a WR-SPM (Mizutani et al., 2007). For this purpose, Madin-Darby canine kidney (MDCK) cells were cultured on a collagen gel substrate and stretched via the deformation of collagen gel substrate. The stiffness map of the collagen gel and the cells was measured before and after the stretch (Figure $3 \mathrm{a}, \mathrm{b})$. Cellular stiffness at intercellular junctions and in other regions of the cells was averaged, and the change in stiffness-before and after the stretch-was also compared (Fig. 3c). Results, showed the junctional site exhibiting a 2-fold increase in the stiffness. In contrast, the stiffness of another region of the cell increased slightly. The local stiffness of each condition was plotted as a histogram, and the frequency of stiffness was fitted to a Gaussian function (Fig. 3d). The stiffness of the collagen gel did not change under the stretch conditions while the stiffness of the cells increased under these conditions. We analyzed the morphological changes in the cells and collagen gel during the uniaxial stretch. The stretch-induced strain fields on the collagen gels and the cells were analyzed as a first approximation (Fig. 3e and Table 1). Some areas of the collagen gel were observed to be homogeneously stretched; the distance between two arbitrary points was increased by $5 \%$ in the direction shown by the arrows in Figure 3e. In contrast, each cell was heterogeneously stretched: the variance of the increments ranged from $4 \%$ to $6 \%$.

We consider two possible reasons for the stretchinduced changes in the stiffness of the cells: it may be due to an elastic response of the actin filaments (Kojima et al., 1994), or it may be attributed to changes in the cellular activities such as enhancement of the contractile force and the reinforcement of stress fibers (Glogauer et al., 1998; Mizutani et al., 2004a). Janmey et al., (1991) investigated the elastic property of an actin matrigel and showed that more than $10 \%$ of the strain was required to yield a 2 -fold increase in stress. In our study, the degree of the strain in the cells ranged from $4 \%$ to $6 \%$ (Table 1 ). Considering these findings, the degree of cellular deformation induced in our study was too small to yield a 2-fold increase in the stiffness of the actin networks. Therefore, the increase in the stiffness may be due to the physiological activities of the epithelial cells.

To our knowledge, no increased stiffness at the intracellular junctions of epithelial cells under stretch conditions (Fig. 3c) has ever been report. It is true that Trepat et al. (2004) examined the viscoelastic moduli of the bulk epithelial sheet under mechanical stretch. They 

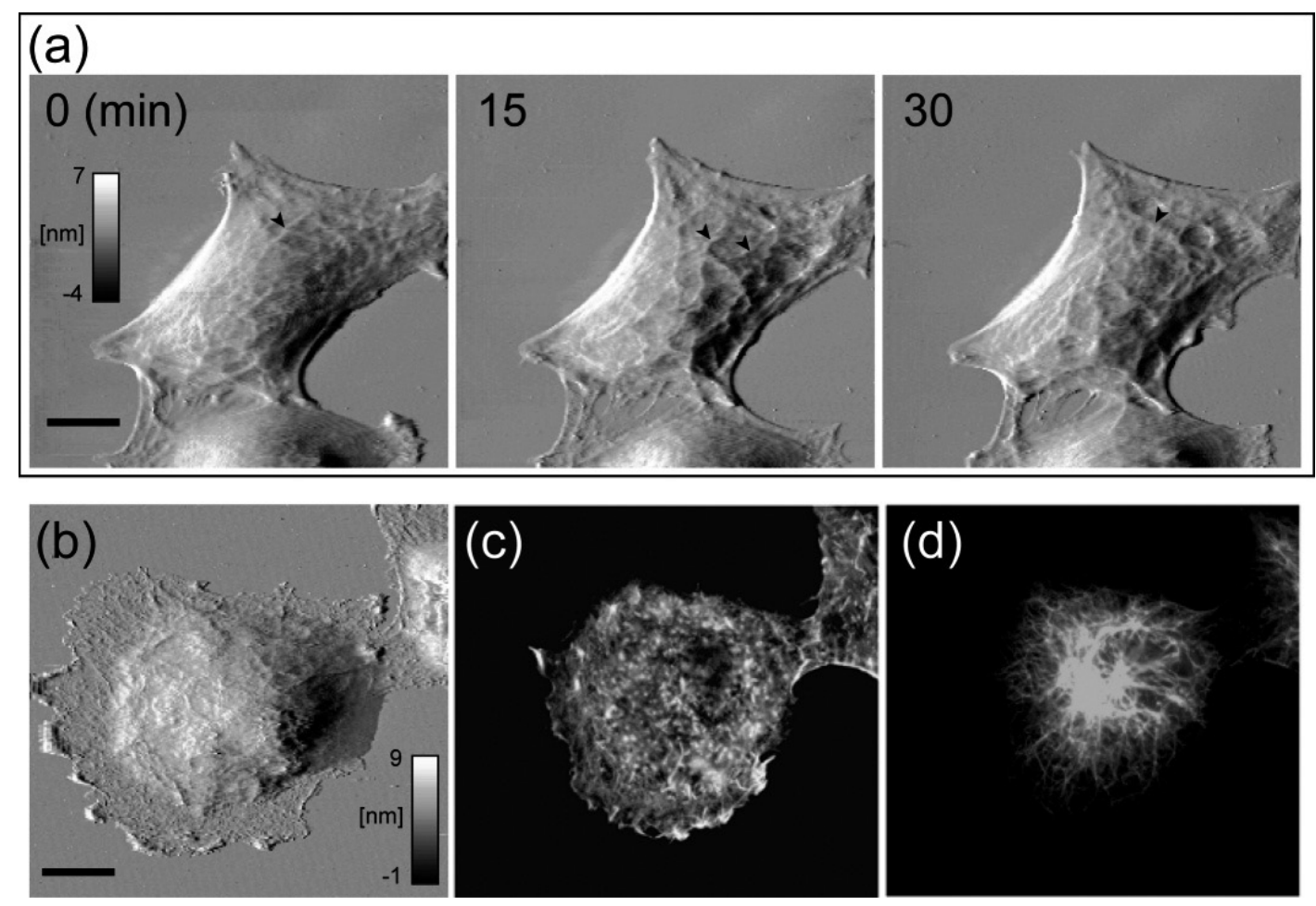

Fig. 4. a: Time-lapse deflection images of living melanoma cells taken every $15 \mathrm{~min}$. The pixel resolusion of these images is $256 \times 256$ pixels. Mesh-like structures observed on the cellular surface are changing dynamically (arrowheads). Bar $=10 \mu \mathrm{m}$. b: A deflection image of a living melanoma cell measured by the constant force mode with the WR-SPM. The data acquisition time was 15 min. c, d: Distributions of F-actin (c) and vimentin (d) near the cellular surface of the identical cell in $\mathbf{b}$. Bar $=10 \mu \mathrm{m}$. Reproduced with permission from Kato et al. (2006) Jpn J Appl Phys 45: 2328-2332.

showed that the cellular storage modulus increased with stretch, but they did not report any increased stiffness at the intercellular junction. Although the physiological implications of increased stiffness at intercellular junctions are not yet known, we presume that this increase would be involved in strengthening the intercellular adhesions to resist an external loading such as mechanical stretch. This hypothesis should be tested in our future studies by observing the recruitment of intercellular adhesion proteins such as cadherins and catenins to the intercellular junctions under a mechanical stretch.

\section{Observation of a mesh structure on the surface of melanoma cells by WR-SPM}

The cortex of the cellular surface is important for the expression of various cellular functions. Especially, the physical property of the cellular cortex - such as rigidity of the cortex-contributes to the normal cell division (Hickson et al., 2006) and maintains shapes of the cell against extra-cellular forces (Mitchison et al., 2008). However, the detailed mechanism of how the rigid cellular cortex contributes to cellular functions is unknown. One possible answer to the guestion is to consider the morphology of the rigid cortex in relation to the cytoskeletal meshworks because the cytoskeletal networks occasionally provide sites for molecular reactions.

In order to analyze the structure of the cellular cortex in relation to its rigidity, we observed the surface morphology of a living melanoma cell (M2 cell) using WR-SPM and compared the morphology and the immuno-fluorescent images of cytoskeletal proteins (Kato et al., 2006). Time-lapse imaging of the melanoma cell by WR-SPM clearly showed the presence of mesh structures on the cell surface (Fig. 4a).The mesh structures changed dynamically within approximately ten 
(a)

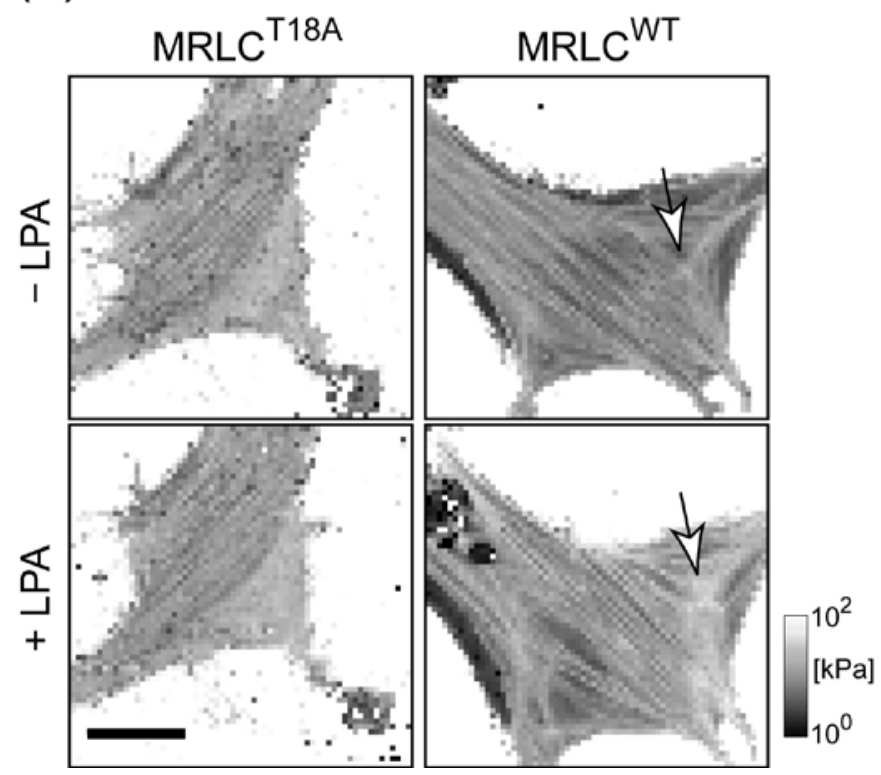

(b)

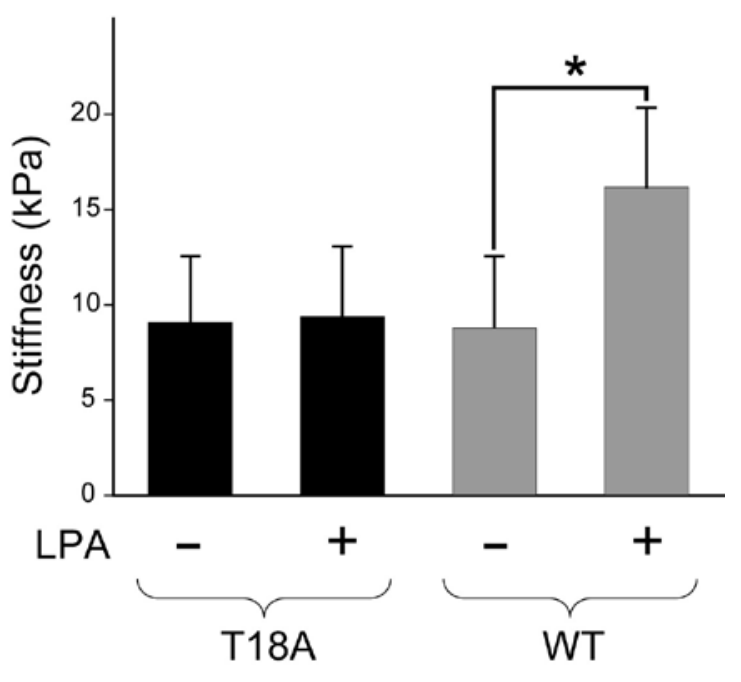

Fig. 5. a: Stiffness map of cells expressing MRLC ${ }^{\mathrm{T} 18 \mathrm{~A}}$ or $M R L C^{\mathrm{WT}}$ after LPA stimulation. Stiffness images were measured by the WR-SPM before and after LPA stimulation. A significant increase in stiffness was observed in cells expressing MRLC ${ }^{\mathrm{WT}}$ after LPA stimulation (arrows). On the other hand, MRLC ${ }^{\mathrm{T} 18 \mathrm{~A}}$ expression suppressed the increase in stiffness after LPA stimulation. The bar denotes a length of $20 \mu \mathrm{m}$. b: Statistical analysis of stiffness in cells expressing MRLC ${ }^{\text {T18A }}$ or MRLC ${ }^{\mathrm{WT}}$. The bar graph shows the averaged stiffness of the cells expressing MRLC ${ }^{\text {T18A }}$ or MRLC ${ }^{\text {WT }}$ before (-) and after (+) LPA stimulation. Data represent mean \pm SE from six experiments $\left({ }^{*} P<0.01\right)$. Reproduced with permission from Mizutani et al. (2006) J cell Physiol 209: 726-731.

minutes. Morphology of the cellular surface (Fig. 4b) was also compared with the distribution of actin (Fig. 4c) and vimentin (Fig. 4d) because actin and vimentin, which are kinds of cytoskeletal proteins, are thought to mechanically support cells (Janmey et al., 1991). However, the mesh structures observed by WR-SPM did not correspond to the distribution of actin and vimentin. This finding indicates that the mesh structures are not related to the stress fibers.

Recently, similar mesh structures were reported in the dorsal surface of bovine pulmonary artery endothelial cells (BPAECs) by SPM measurements (Pesen and Hoh, 2005). The results showed that F-actin and vimentin are major contributors to the mesh structures in the case of BPAECs. The discrepancy between the previous report and our finding should be clarified by further studies.

\section{Measurement of the regulatory mechanism of the cellular contractile force by WR-SPM}

The cellular contractile force plays a ceutral role in the performance of various physiological cellular functions such as cell movement, cytokinesis, and maintenance of the cell morphology. The main generators of the contractile force mainly originates from the interaction between actin filaments and myosin II, in which the phosphorylation of the myosin II regulatory light chain (MRLC) is necessary (Ikebe et al., 1986; Somlyo and Somlyo, 2003). Two phosphorylatable sites - threonine 18 and serine 19 - are responsible for the activation of the myosin motor activity of MRLC. Serine 19 is more easily phosphorylated than threonine 18, allowing MRLC to be in either a de-, mono-, or diphosphorylated state (Ikebe et al., 1986). Previous in vitro motility assay showed that myosin II with diphosphorylated MRLC (PP-MRLC) has a higher actin-activated $\mathrm{Mg}^{2+}$-ATPase 
Fig. 6. Topography and Stiffness map of a wild type NIH-3T3 cell after LPA stimulation. Topography and stiffness images were measured before ( $(-)$ and after (+) LPA stimulation. The cell stiffened. Bar $=30 \mu \mathrm{m}$
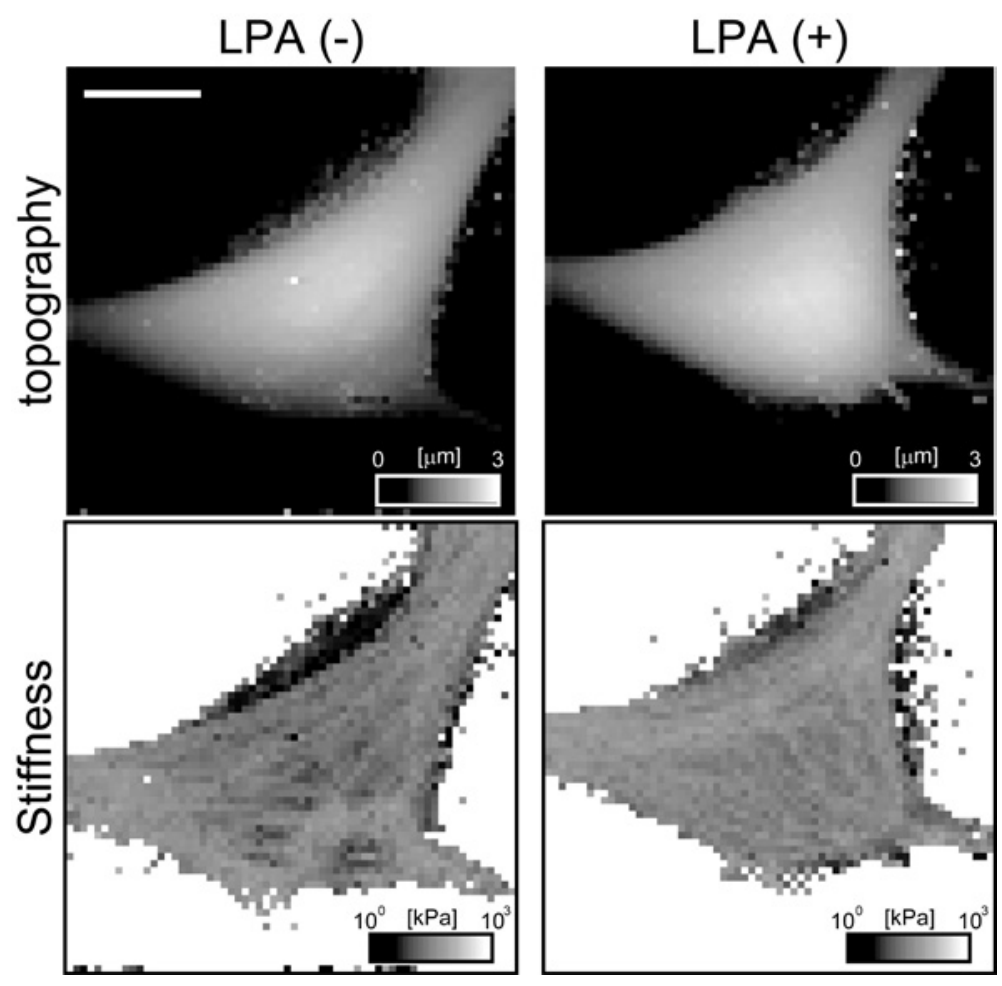

activity than that with monophosphorylated MRLC (P-MRLC) (Ikebe et al., 1988; Umemoto et al., 1989). Although several biochemical differences between the in vitro phosphorylated MRLCs were reported (Colburn et al., 1988; Trybus, 1989), a clear distinction between the phosphorylated MRLCs in the generation of the contractile force has not been determined.

To examine whether PP-MRLC brings a higher in vivo contractile force than P-MRLC, we measured the stiffness of cells expressing wild-type MRLC (MRLC ${ }^{\mathrm{WT}}$ ) cells and a mutant type MRLC (MRLC ${ }^{\mathrm{T} 18 \mathrm{~A}}$ ) cellsbefore and after treatment with an activator of MRLC phosphorylation (LPA; lysophosphatidic acid) (Mizutani et al., 2006). Stiffness maps of the cells expressing MRLC ${ }^{\mathrm{T} 18 \mathrm{~A}}$ or MRLC ${ }^{\mathrm{WT}}$ were shown in Figure 5a. Stiffer fibrous structures corresponding to the stress fibers were observed in both images. The MRLC ${ }^{\mathrm{T} 18 \mathrm{~A}}$ expressing cells, did not change the in cellular stiffness under LPA stimulation, while the MRLC ${ }^{\mathrm{WT}}$-expressing cells had stiffened after LPA stimulation. The regions indicated by the arrows in Figure 5a had especially stiffened after LPA stimulation. The cellular stiffness in each condition was averaged to the degree of change in stiffness due to the LPA treatment (Fig. 5b). There was a significant difference in cellular stiffness between MRLC $^{\text {T18A }}$ - and MRLC ${ }^{\mathrm{WT}}$-expressing cells after LPA treatment although there was little difference between these cells before LPA stimulation. We conclude that the diphosphorylation of MRLC enhances the intracellular tension.

The increase of cellular stiffness by LPA stimulation may be due not only to the diphosphorylatoin of MRLC but also to the increment of the number of stress fibers. However, we consider the latter is unlikely because of the following reason. It is proposed that the transition from the dephosphorylation to monophosphorylation of MRLC might induce the formation of new stress fibers. Our results demonstrate that MRLC ${ }^{\mathrm{T} 18 \mathrm{~A}}$ - expressing cells did not increase their stiffness at the cell center despite the cell's capability of constructing additional stress fibers. On the other hand, MRLC ${ }^{\mathrm{WT}}$-expressing cells increased their stiffness in the central area. Furthermore, a previous report showed that, in cells stimulated by calyculin A (a serine/threonine phosphatase inhibitor), the amount of P-MRLC increased at the cell periphery but was unchanged at the center (Peterson et al., 2004). Taken together into consideration, these findings imply that stress fibers are not formed at the center of the cell by 
LPA stimulation; instead, these results strongly suggest that the transition from mono- to diphosphorylated MRLC enhances the tension acting on stress fibers.

\section{Conclusion}

In the present study, we introduced a wide range scanning probe microscope (WR-SPM) which is specialized to measure a sample for $400 \mu \mathrm{m}$ in the $x y$ directions and $23 \mu \mathrm{m}$ in the $z$ direction. We showed this WR-SPM to be useful for studies of the shape and surface structure of living cultivated cells in a liquid environment. We also demonstrated that the combination of topographical and mechanical modes of SPM is a powerful technique for analyzing the mechanical properties of living cells in relation to the morphology of the cell. Thus, the WR-SPM is expected to be widely used in biophysical fields for analyzing the function of living cells.

\section{References}

Benjamin M, Hillen B Mechanical influences on cells, tissues and organs- 'Mechanical Morphogenesis'. Eur J Morphol 41: 3-7 (2003).

Colburn JC, Michnoff CH, Hsu LC, Slaughter CA, Kamm KE, Stull JT: Sites Phosphorylated in Myosin Light Chain in Contracting Smooth-Muscle. J Biol Chem 263: 19166-19173 (1988).

Engler AJ, Sen S, Sweeney HL, Discher DE: Matrix elasticity directs stem cell lineage specification. Cell 126: 677-689 (2006).

Glogauer M, Arora P, Chou D, Janmey PA, Downey GP, McCulloch CAG: The role of actin-binding protein 280 in integrin-dependent mechanoprotection. J Biol Chem 273: 1689-1698 (1998).

Haga H, Sasaki S, Kawabata K, Ito E, Ushiki T, Sambongi T: Elasticity mapping of living fibroblasts by AFM and immunofluorescence observation of the cytoskeleton. Ultramicroscopy 82: 253-258 (2000).

Hickson GRX, Echard A, O'Farrell PH: Rho-kinase controls cell shape changes during cytokinesis. Current Biol 16: 359-370 (2006).

Ikebe M, Hartshorne DJ, Elzinga M: Identification, phosphorylation, and dephosphorylation of a 2 nd site for myosin light chain kinase on the 20,000-dalton light chain of smooth-muscle myosin. J Biol Chem 261: 36-39 (1986).
Ikebe M, Koretz J, Hartshorne DJ: Effects of phosphorylation of light chain residues threonine-18 and serine-19 on the properties and conformation of smooth-muscle myosin. J Biol Chem 263: 6432-6437 (1988).

Janmey PA, Euteneuer U, Traub P, Schliwa M: Viscoelastic properties of vimentin compared with other filamentous biopolymer networks. J Cell Biol 113: 155-160 (1991).

Kato K, Ohmori Y, Mizutani T, Haga H, Ohashi K, Ito T, Kawabata $\mathrm{K}$ : The role of actin-binding protein filamin A in cellular stiffness and morphology studied by widerange scanning probe microscopy. Jpn J Appl Phys Part 1 45: 2328-2332 (2006).

Kljaer M: Role of extracellular matrix in adaptation of tendon and skeletal muscle to mechanical loading. Physiol Rev 84: 649-698 (2004).

Kojima H, Ishijima A, Yanagida T: Direct measurement of stiffness of single actin-filaments with and without tropomyosin by in-vitro nanomanipulation. Proc Natl Acad Sci USA 91: 12962-12966 (1994).

Lo CM, Wang HB, Dembo M, Wang YL: Cell movement is guided by the rigidity of the substrate. Biophys $J$ 79: 144-152 (2000).

Mierke CT, Rosel D, Fabry B, Brabek J: Contractile forces in tumor cell migration. Eur J Cell Biol 87: 669676 (2008).

Mitchison TJ, Charras GT, Mahadevan L: Implications of a poroelastic cytoplasm for the dynamics of animal cell shape. Semin Cell Devel Biol 19: 215-223 (2008).

Mizutani T, Haga H, Kawabata K: Cellular stiffness response to external deformation: Tensional homeostasis in a single fibroblast. Cell Motil Cytoskelet 59: 242-248 (2004a).

Mizutani T, Haga H, Nemoto K, Kawabata K: Wide-range scanning probe microscopy for visualizing biomaterials in the submillimeter range. Jpn J Appl Phys Part 143 : 4525-4528 (2004b).

Mizutani T, Haga H, Koyama Y, Takahashi M, Kawabata $\mathrm{K}$ : Diphosphorylation of the myosin regulatory light chain enhances the tension acting on stress fibers in fibroblasts. J Cell Physiol 209: 726-731 (2006).

Mizutani T, Haga H, Kawabata K: Development of a device to stretch tissue-like materials and to measure their mechanical properties by scanning probe microscopy. Acta Biomaterial 3: 485-493 (2007).

Nagayama M, Haga H, Takahashi M, Saitoh T, Kawabata $\mathrm{K}$ : Contribution of cellular contractility to spatial and temporal variations in cellular stiffness. Exp Cell Res 300: 396-405 (2004). 
Pesen D, Hoh JH: Micromechanical architecture of the endothelial cell cortex. Biophys J 88: 670-679 (2005).

Peterson LJ, Rajfur Z, Maddox AS, Freel CD, Chen Y, Edlund M, Otey C, Burridge K: Simultaneous stretching and contraction of stress fibers in vivo. Mol Biol Cell 15: 3497-3508 (2004).

Somlyo AP, Somlyo AV: $\mathrm{Ca}^{2+}$ sensitivity of smooth muscle and nonmuscle myosin II: Modulated by G proteins, kinases, and myosin phosphatase. Physiol Rev 83: 1325-1358 (2003).

Trepat X, Grabulosa M, Puig F, Maksym GN, Navajas D, Farre R: Viscoelasticity of human alveolar epithelial cells subjected to stretch. Am J Physiol Lung Cell Mol Physiol 287: L1025-L1034 (2004).

Trybus KM: Filamentous smooth-muscle myosin is regulated by phosphorylation. J Cell Biol 109: 28872894 (1989).
Umemoto S, Bengur AR, Sellers JR: Effect of multiple phosphorylations of smooth-muscle and cytoplasmic myosins on movement in an Invitro motility assay. $J$ Biol Chem 264: 1431-1436 (1989).

vanEijden TMGJ, Koolstra JH, Brugman P: Threedimensional structure of the human temporalis muscle. Anat Rec 246: 565-572 (1996).

Wiskott HWA, Belser UC: Lack of integration of smooth titanium surfaces: a working hypothesis based on strains generated in the surrounding bone. Clin Oral Implants Res 10: 429-444 (1999).

Yeung T, Georges PC, Flanagan LA, Marg B, Ortiz M, Funaki M, Zahir N, Ming WY, Weaver V, Janmey PA: Effects of substrate stiffness on cell morphology, cytoskeletal structure, and adhesion. Cell Motil Cytoskelet 60: 24-34 (2005) 